OPEN ACCESS

Edited by:

Tong-Chuan $\mathrm{He}$,

University of Chicago Medicine,

United States

Reviewed by:

Amar Bennasroune,

Université de Reims Champagne-

Ardenne, France

Deyao Shi,

Huazhong University of Science and

Technology, China

Jianxiang Liu,

Huazhong University of Science and

Technology, China

*Correspondence:

Shenglong $L$

lishenglong@cancerhosp-In-cmu.com

Specialty section:

This article was submitted to Pharmacology of Anti-Cancer Drugs,

a section of the journal

Frontiers in Oncology

Received: 06 February 2021

Accepted: 22 April 2021

Published: 20 May 2021

Citation:

Li S (2021) Anlotinib:

A Novel Targeted Drug for

Bone and Soft Tissue Sarcoma.

Front. Oncol. 11:664853.

doi: 10.3389/fonc.2021.664853

\section{Anlotinib: A Novel Targeted Drug for Bone and Soft Tissue Sarcoma}

\author{
Shenglong $L i^{1,2^{*}}$ \\ ${ }^{1}$ Department of Bone and Soft Tissue Tumor Surgery, Cancer Hospital of China Medical University, Liaoning Cancer \\ Hospital \& Institute, Shenyang, China, ${ }^{2}$ Department of Tissue Engineering, Center of 3D Printing \& Organ Manufacturing, \\ School of Fundamental Sciences, China Medical University (CMU), Shenyang, China
}

Bone and soft tissue sarcomas account for approximately $15 \%$ of pediatric solid malignant tumors and $1 \%$ of adult solid malignant tumors. There are over 50 subtypes of sarcomas, each of which is notably heterogeneous and manifested by remarkable phenotypic and morphological variability. Anlotinib is a novel oral tyrosine kinase inhibitor (TKI) targeting c-kit, platelet-derived growth factor receptors, fibroblast growth factor receptor, and vascular endothelial growth factor receptor. In comparison with the placebo, anlotinib was associated with better overall survival and progression-free survival (PFS) in a phase III trial of patients with advanced non-small cell lung cancer (NSCLC), albeit with cancer progression after two previous lines of treatment. Recently, the National Medical Products Administration approved anlotinib monotherapy as a thirdline treatment for patients with advanced NSCLC. Additionally, a phase IIB randomized trial substantiated that anlotinib is associated with a significant longer median PFS in patients with advanced soft tissue sarcoma. Moreover, anlotinib is also effective in patients with advanced medullary thyroid carcinoma and metastatic renal cell carcinoma. Anlotinib has similar tolerability to other TKls targeting vascular endothelial growth factor receptors and other tyrosine kinase-mediated pathways. However, anlotinib has a notably lower rate of side effects $\geq$ grade 3 relative to sunitinib. This review discussed the remarkable characteristics and major dilemmas of anlotinib as a targeted therapy for sarcomas.

Keywords: anlotinib, targeted therapy, sarcoma, anti-angiogenesis, multidrug resistance

\section{INTRODUCTION}

Bone and soft tissue sarcoma (STS) is a class of tumors in the leaf system, including primary malignant bone tumor and STS $(1,2)$, accounting for approximately $1 \%$ of adult and $15 \%$ of pediatric malignant tumors (3). The three most prevalent primary malignant bone tumors are Ewing's sarcoma, chondrosarcoma, and osteosarcoma. STS is pathologically complex and has more

\footnotetext{
Abbreviations: TKI, tyrosine kinase inhibitor; OS, overall survival; PFS, progression-free survival; NSCLC, non-small cell lung cancer; STS, soft tissue sarcoma; mRCC, metastatic renal cell carcinoma; TA, tumor angiogenesis; VEGF, vascular endothelial growth factor; PDGFR, platelet-derived growth factor receptors; VEGFR, vascular endothelial growth factor receptors; FGFR, fibroblast growth factor receptors; HUVECs, human umbilical vein endothelial cells; PK, pharmacokinetics; DDP, cisplatin; SCLC, small cell lung cancer; GDNF, glial cell line-derived neurotrophic factor; MAPK, mitogen-activated protein kinase; PLC $\gamma$, phospholipase $\gamma 1$; PKC, protein kinase C; RAS, rat sarcoma protein; SCF, stem-cell factor; t1/2, elimination half-life; AE, adverse event; WDLS, well-differentiated liposarcoma; DDLS, dedifferentiated liposarcoma.
} 
than 100 subtypes, the most common of which include undifferentiated pleomorphic sarcoma, liposarcoma, and leiomyosarcoma (4-8) (Figure 1). Prior to the introduction of chemotherapies, the long-term survival rate was only $20-40 \%$ in patients with bone sarcoma and only $35 \%$ in patients with STS $(9,10)$. Since the 1970s, chemotherapies have been associated with significantly better outcomes for sarcoma, and the five-year survival rate is $60-80 \%$ in patients treated using chemotherapies and surgical resection. Only surgical resection may cure sarcomas, albeit with significantly better outcomes when combined with chemotherapies $(9,11)$. Approximately $10 \%$ of patients with sarcomas are also detected with metastatic lesions (12). Moreover, metastatic diseases occur in $25 \%$ of patients with sarcomas after the radical treatment of primary tumors. There is an urgent need for new treatments for sarcomas $(12,13)$. However, there are several limitations for the development and investigation of new treatments, such as the presence of various tumor subtypes, small available sample sizes, and heterogeneous patient populations.

New vessels in tumor tissues are lifelines for the development of tumor cells. Through continuous angiogenesis, nutrients are continuously supplied and, at the same time, provide vascular channels for the distant local invasion and metastasis of tumor cells (14-16). Therefore, continuous angiogenesis ensures the continuation of the biological behaviors of tumors $(15,16)$. Tumor angiogenesis (TA) also plays an essential role in tumors, especially in the malignant tumor system. Many cytokines, including stimulating factors and inhibiting factors, control and regulate this process $(17,18)$. The balance between these two cytokines is dependent on the genetic structures of tumor cells, mesenchymal components, and intratumoral metabolic environment (19). It is certain that TA and its important roles in tumor occurrence and progression affect the diagnosis and treatment of malignant tumors. Body tissues and organs, including tumor growths, must be supplied with oxygen and nutrients through blood vessels. Meanwhile, malignant tumor cells may also enter their surrounding tumor vessels and spread to distant places $(20,21)$. Therefore, TA is vital in each step of the occurrence and development of tumors, including growth, infiltration, and metastasis. The occurrence and development of TA is complex and rigorous. Vascular endothelial growth factor (VEGF) is expressed in many tumors as an important regulatory factor in TA (22-25). VEGF is synthesized and secreted by both normal and tumor cells and is highly expressed in most malignant tumors, and may induce TA and promote tumor growth, metastasis, and invasion $(22,23)$. Normal tissue VEGF is lowly and stably expressed. In consideration of the effects on angiogenesis and that overexpressed VEGF receptors (VEGFRs; especially VEGFR-2) correlate with a low survival rate in patients with sarcoma, these patients can benefit from VEGF/VEGFR targeted therapy (26-28).

Anlotinib (AL3818) is a new oral multi-target tyrosine kinase inhibitor (TKI) with extensive anticancer activity in various solid tumors in vivo and in vitro (29-38). Anlotinib suppresses TA and proliferation by blocking the tyrosine kinase receptors in the signaling pathways of stem cell factor receptors, platelet-derived growth factor receptors (PDGFR) $\alpha$ and $\beta$, VEGFRs $1-3$, and fibroblast growth factor receptors (FGFRs) 1-4 (39). Previous reviews have examined the rationale, clinical evidence, and future perspectives of anlotinib for the treatment of multiple cancers (40). However, the role of anlotinib in sarcoma remains uncertain. Therefore, this review provides an overview of anlotinib as a targeted therapy in patients with sarcoma. Firstly, the data from preclinical and clinical studies on anlotinib as a targeted therapy for sarcomas were collected and summarized. Subsequently, we extracted the studies that

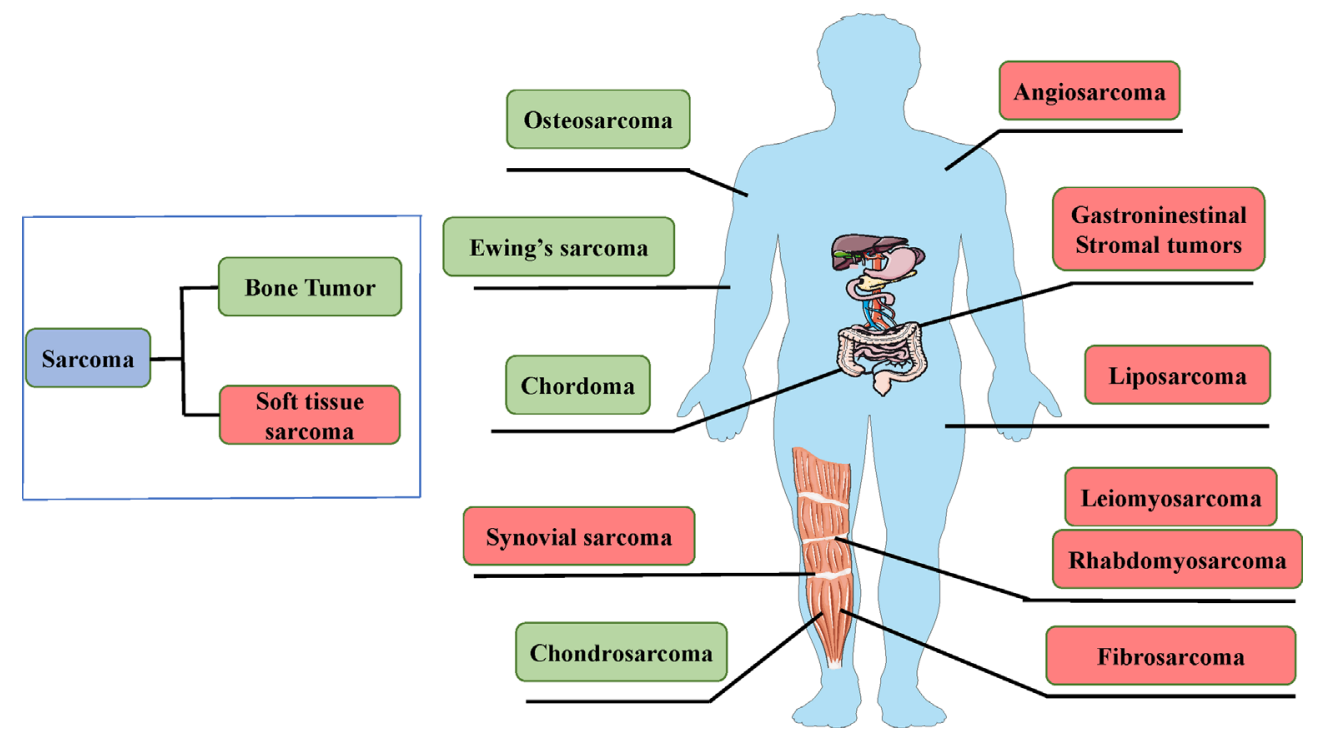

FIGURE 1 | The main sarcoma types are bone tumors and soft tissue sarcomas. 
analyzed the remarkable characteristics of anlotinib as a targeted therapy relative to other anti-angiogenic agents. Finally, this review discusses the ongoing clinical trials, main difficulties, and future directions regarding anlotinib hydrochloride as a targeted therapy for advanced sarcomas.

\section{ANLOTINIB: A NOVEL INHIBITOR TARGETING MULTIPLE RTKS}

Anlotinib was developed by Nanjing Chia Tai Tianqing Pharmaceutical Co., Ltd. as a new oral molecular RTK inhibitor; it targets VEGFR1, VEGFR3, VEGFR2/KDR, PDGFR- $\alpha$, c-Kit, and FGFRs 1-3 and inhibits TA and tumor cell proliferation (31, 41-43). Anlotinib may inhibit more targets than that do other RTK inhibitors, such as pazopanib, sunitinib, and sorafenib. All targets of anlotinib and other RTK inhibitors are shown in Table 1.

The main mechanisms of action of anlotinib are as follows: preclinical studies have shown that anlotinib inhibits VEGF/ PDGF-BB/FGF-2-induced cell migration, angiogenesis, and capillary-like tube formation in endothelial cells $(44,45)$. More specifically, the mechanism involves the inhibition of the downstream ERK signaling pathway. Anlotinib has stronger anti-angiogenesis activity than that do other antiangiogenic agents (sunitinib and sorafenib) (46). Moreover, anlotinib can bind to VEGFR2 tyrosine kinase ATP binding pocket and highly selectively inhibit VEGFR2 $\left(\mathrm{IC}_{50}<1 \mathrm{nmol} / \mathrm{L}\right)$, which suppresses the proliferation of human umbilical vein endothelial cells (HUVECs). In addition, anlotinib reduces blood vessel density in vivo and suppresses HUVEC migration, microvascular growth, and angiopoiesis in vitro. Anlotinib has more extensive and better anti-tumor responses than those by sunitinib in vivo (31). For cell lines that express mutant FGFR2 proteins, anlotinib can exert a strong inhibition effect on cell growth. However, similar to other oral RTK inhibitors, the combination of anlotinib with carboplatin and paclitaxel is less effective than that is anlotinib alone (47) (Figures 2 and 3).

\section{PHARMACOKINETICS AND TOLERABILITY OF ANLOTINIB}

Studies of animals and patients with advanced solid tumors have assessed the PK characteristics of anlotinib $(39,48)$. PK and drug

TABLE 1 | The different targets between anlotinib and other RTK. inhibitors.

\begin{tabular}{|c|c|c|c|c|c|c|c|c|c|c|}
\hline & \multicolumn{3}{|c|}{ VEGFR } & \multicolumn{2}{|c|}{ PDGFR } & \multicolumn{4}{|c|}{ FGFR } & \multirow[t]{2}{*}{ Others } \\
\hline & 1 & 2 & 3 & $\alpha$ & $\beta$ & 1 & 2 & 3 & 4 & \\
\hline Anlotinib & $\sqrt{ }$ & $\sqrt{ }$ & $\sqrt{ }$ & $\sqrt{ }$ & $\sqrt{ }$ & $\sqrt{ }$ & $\sqrt{ }$ & $\sqrt{ }$ & $\sqrt{ }$ & $\mathrm{C}-\mathrm{KIT}(+)$ \\
\hline Pazopanib & $\sqrt{ }$ & $\sqrt{ }$ & $\sqrt{ }$ & $\sqrt{ }$ & $\sqrt{ }$ & $\sqrt{ }$ & $\sqrt{ }$ & $\times$ & $\times$ & $\mathrm{C}-\mathrm{KIT}(+)$ \\
\hline Nintedanib & $\sqrt{ }$ & $\sqrt{ }$ & $\sqrt{ }$ & $\sqrt{ }$ & $\sqrt{ }$ & $\sqrt{ }$ & $\sqrt{ }$ & $\sqrt{ }$ & $x$ & FLT3(+), $\operatorname{Src}(+)$ \\
\hline Vatalanib & $\sqrt{ }$ & $\sqrt{ }$ & $\sqrt{ }$ & $x$ & $\sqrt{ }$ & $x$ & $x$ & $x$ & $x$ & $\mathrm{C}-\mathrm{KIT}(+)$ \\
\hline Axitinib & $\sqrt{ }$ & $\sqrt{ }$ & $\sqrt{ }$ & $x$ & $x$ & $x$ & $x$ & $x$ & $x$ & $x$ \\
\hline Sunitinib & $\sqrt{ }$ & $\sqrt{ }$ & $\sqrt{ }$ & $\sqrt{ }$ & $\sqrt{ }$ & $x$ & $x$ & $\times$ & $\times$ & FLT3(+), c-KIT(+), RET(+), CSF1R(+) \\
\hline Sorafenib & $\sqrt{ }$ & $\sqrt{ }$ & $\sqrt{ }$ & $x$ & $\sqrt{ }$ & $x$ & $x$ & $x$ & $x$ & RET (+), c-KIT(+), FLT3(+) \\
\hline
\end{tabular}

$\sqrt{ }=$ target,$\times=$ no target.

\section{Chemical structure of anlotinib}

FIGURE 2 | The chemical structure of anlotinib. 


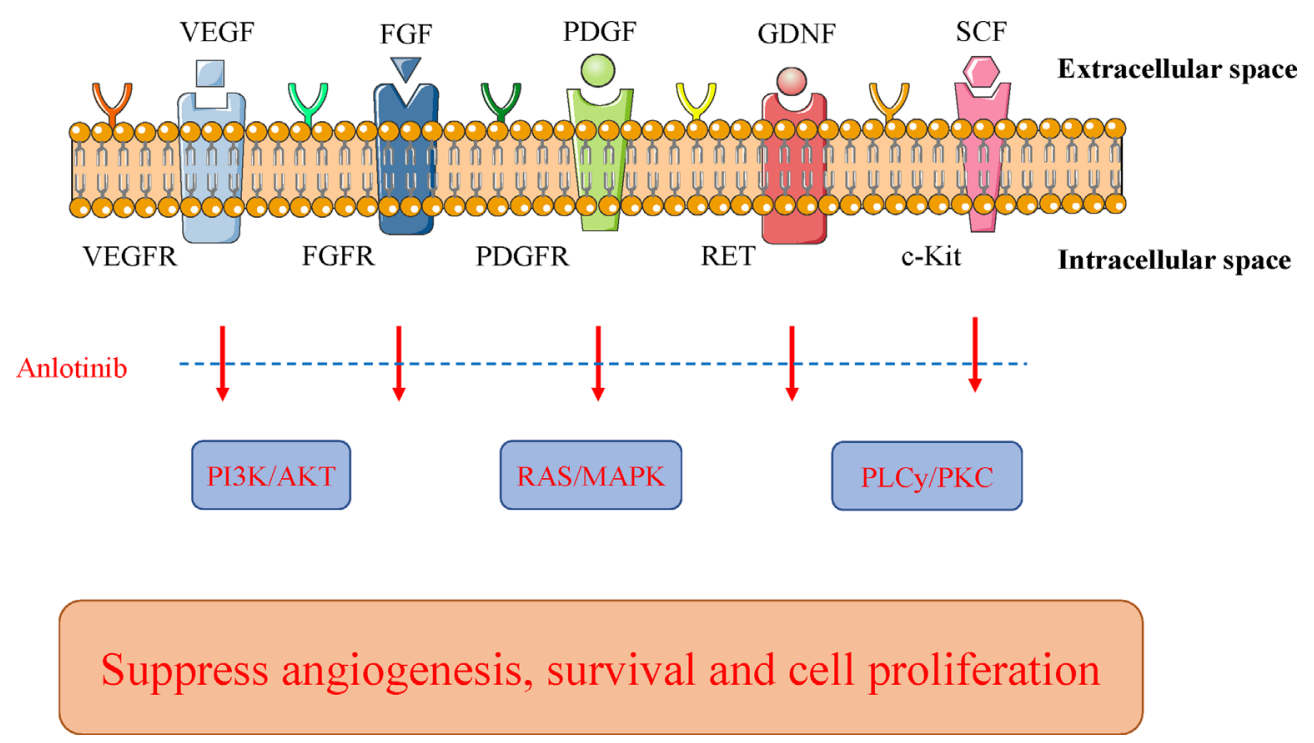

FIGURE 3 | Mechanism of action of anlotinib. Anlotinib can suppress tumor cell growth through some key pathways, including PI3K/AKT, RAS/MAPK, and PLCy/ PKC. Their key receptors include VEGFR, FGFR, and PDGFR. Anlotinib blocks the activated signals from these oncogenic receptors to the key pathways. FGFR, fibroblast growth factor receptor; GDNF, glial cell line-derived neurotrophic factor; MAPK, mitogen-activated protein kinase; PDGFR, platelet-derived growth factor receptor; PLC $\gamma$, phospholipase $\gamma 1$; PKC, protein kinase C; RAS, rat sarcoma protein; SCF, stem-cell factor; VEGFR, vascular endothelial growth factor receptor.

preparation studies have revealed satisfactory membrane permeability and absorption rates with anlotinib. The oral bioavailability of anlotinib was $41-77 \%$ and $28-58 \%$ in canines and rats, respectively, with significantly different biotransformation rates in different species. Anlotinib had a high apparent volume of distribution in rats $(27.6 \pm 3.1 \mathrm{~L} / \mathrm{kg})$ and canines $(6.6 \pm 2.5 \mathrm{~L} / \mathrm{kg})$ in vivo. In all species, including rats (97\%), canines (96\%), and humans (93\%), anlotinib was highly bound to the plasma. In human plasma, anlotinib could mainly bind to albumins and lipoproteins. Anlotinib showed significantly higher levels in the tissues of tumor-bearing mice and rats than those in plasma (48). Multiple human cytochrome P450 subtypes could metabolize anlotinib in vitro mainly because of CYP3A4 and CYP3A5; this indicates that circulating anlotinib levels and their effects may be influenced by liver drugs that affect P450 enzyme functions (48). Anlotinib showed notable induction effects on CYP2D1 and CYP3A1/2 in vivo. However, after oral administration in rats, anlotinib had insignificant induction effects on CYP1A2, CYP2D2, and CYP2C6. Therefore, attention should be paid to when anlotinib is used with other CYP2D1 and CYP3A1/2 metabolized drugs (49). The PK characteristics of anlotinib have been subjected to two phase I clinical trials. In a phase I clinical study in China, most patients showed significantly higher plasma anlotinib concentrations $1 \mathrm{~h}$ after administration, demonstrating that anlotinib can be rapidly absorbed by the intestinal tract. The Cmax and AUC0-120 h of anlotinib $120 \mathrm{~h}$ after administration increased from $5 \mathrm{mg} /$ person to $16 \mathrm{mg} /$ person with dosage; however, the proportion of dosage was uncertain (39). At a dose of $16 \mathrm{mg} /$ person of anlotinib, the mean Cmax was $10.5 \pm 2.9 \mathrm{ng} / \mathrm{ml}$. The Tmax of anlotinib after administration was $4-11 \mathrm{~h}$, while the elimination half-life (t1/2) was $96 \pm 17 \mathrm{~h}$ (27716285). Anlotinib showed considerably longer $\mathrm{t} 1 / 2$ in patients relative to that by most TKIs used in clinical settings to date (i.e., 3-60 h) (50). Such longer $\mathrm{t} 1 / 2$ resulted in notable plasma anlotinib accumulation over time, and the mean accumulation ratio was $12 \pm 7$. The two-week subchronic administration regimen led to a consecutive increase in the plasma concentration of anlotinib, which reached its peak at 14 days. Subsequently, plasma anlotinib was reduced in a seven-day washout period. Based on these results and the toxicity descriptions, this phase I study suggested that the administration regimen in future studies should be $12 \mathrm{mg}$ every day for 12 weeks continuously, followed by a one week rest period (39).

Using PK data, anlotinib tolerability has been investigated in different cancer studies. A dosage of $12 \mathrm{mg}$ (qd for two weeks continuously, followed by one week of rest) is the recommended regimen in phase I-III clinical trials. In phase I trials, all adverse events (AEs) appeared to be controllable. The incidence of the most common AEs was more than $30 \%$, including hypertension (34\%), proteinuria $(67 \%)$, hand-foot skin reaction $(53 \%)$, hypothyroidism (57\%), elevated alanine aminotransferase (48\%), elevated aspartate transaminase $(43 \%)$, elevated total bilirubin $(38 \%)$, elevated triglyceride $(62 \%)$, elevated total cholesterol $(62 \%)$, serum amylase $(43 \%)$, abnormal myocardial enzyme (38\%), neutropenia (33\%), and leukopenia (33\%) (27716285). Grade 3/4 AEs were reported in 29\% patients, including elevated lipase (5\%), hypertension (10\%), hand-foot skin reaction $(5 \%)$, and elevated triglyceride (10\%) (39). Anlotinib induced lesser and milder diarrhea relative to that by other oral anti-VEGFR TKIs (51-53). Importantly, the incidence of elevated triglyceride and cholesterol was high in anlotinib-treated patients (54). 


\section{PRECLINICAL STUDIES ON ANLOTINIB}

Baseline studies have focused on the assessment of the anti-tumor effect of anlotinib in vitro and in vivo. Anlotinib suppresses cell vitality and induces the apoptosis of human lung cancer cells in vitro, which enhances the cytotoxicity and anti-angiogenesis effects of anlotinib via the JAK2/STAT3/VEGFA signal (55). Anlotinib exerts antitumor effects by suppressing the metastasis, angiogenesis, and cell growth via double blocking MET and VEGFR2 signaling pathways (56). Lin et al. (46) demonstrated that anlotinib inhibits angiogenesis by suppressing the activation of VEGFR2, PDGFR $\beta$, and FGFR1 and the downstream ERK signaling pathway. In addition, at the same concentration, anlotinib has a superior antiangiogenic activity to sunitinib, sorafenib, and nintedanib (46). As encouraged by the inhibitory effects of anlotinib on a variety of cancer cells, potential in vivo anti-tumor activity has been studied using anlotinib alone or in combination with chemotherapy in human xenograft tumor models of multiple cancers (55-57).

In combination with chemotherapy, Wang et al. (56) reported that at a low concentration $(1 \mu \mathrm{M})$, anlotinib promoted cisplatin (DDP)-induced cell apoptosis and increased the inhibitory effects of DDP on the proliferation of osteosarcoma cells. In comparison with anlotinib or DDP only, anlotinib combined with DDP notably reduced tumor weight and volume in vivo (56). These results revealed that anlotinib increased the in vivo and in vitro sensitivity of osteosarcoma cells to DDP. The activation of the FGFR signaling pathway promoted chemotherapy resistance (5861). Anlotinib can target FGFR1-4 and hence, enhance the responses to chemotherapy. Additionally, its wide targeting range may help overcome the drug resistance caused by previous chemotherapy or targeted therapy treatments.

\section{CLINICAL TRIALS OF ANLOTINIB IN ADVANCED STS AND OSTEOSARCOMA}

Recently, more targeted drugs have shown satisfactory effectiveness in patients with certain histological patterns of advanced STS, including ALK inhibitors (e.g., ceritinib and crizotinib), antiPDGFRs (e.g., olaratumab), multi-targeted kinase inhibitors (e.g., imatinib, pazopanib, sorafenib, and sunitinib), and anti-angiogenic drugs (e.g., bevacizumab). Since the approval of pazopanib, a number of other TKIs have entered clinical trials to evaluate whether their activity in STS matches the promising results seen in other solid tumors. Previously, the emerging role of TKIs in the evolving landscape of sarcoma treatment was reviewed (62). However, for the second-line treatment of STS, FDA-approved pazopanib is typically used. STS is relative rare, accounting for approximately $1 \%$ of all solid malignant tumors $(1,2)$. In recent years, new treatments, including eribulin, trabectedin, and pazopanib, have been developed. However, these drugs have not been cleared for the treatment of STS in China. Additionally, it is difficult to obtain much data for treatment guidance because of the rarity of such diseases and because there are many subtypes. The prognosis of such diseases remains poor, with the median overall survival (OS) only just exceeding one year $(63,64)$. The phase I study of Sun et al. (65) revealed that anlotinib has good anti-STS tumor potential. Based on these results, the multi-center phase II study of Chi et al. (66) (NCT01878448) investigated the anlotinib monotherapy in STS patients with disease progression after first-line chemotherapy with anthracyclines. A total of 166 patients were included in the final analysis and participants were administered oral anlotinib once a day (12 $\mathrm{mg}$ per dose for one or two weeks). For 12 weeks, the progression-free rate was $68 \%$ for the primary endpoint and 13\% (95\% CI, 7.6-18.0\%) for Albright's syndrome; the median progression-free survival (PFS) was 5.6 months and OS was 12.0 months. Some STS histological types, such as ASPS, fibrosarcoma, synovial sarcoma, and liposarcoma, were more sensitive to anlotinib, with a PFR-12 weeks of more than $70 \%$. For liposarcoma, the median PFS, OS, and PFR-12 weeks were 5.6 months, 13.0 months, and 63\%, respectively, with good clinical value. Anlotinib was significantly associated with a longer median PFS of ASPS (21 months), suggesting its considerable benefits. The study group further investigated the age, previous treatment methods, and relationship between dose adjustment and efficacy of anlotinib for the treatment of patients with advanced STS through a randomized IIB phase trial (ALTER0203, NCT02449343) of 158 patients. The results revealed that the median PFS of anlotinib-treated patients was similar to that of patients who received no or one previous treatment $(6.70$ vs. 6.33 months, respectively). The median PFS of the patients $<65$ years old was similar to that of patients $\geq 65$ years old $(6.33 v s .5 .90$ months, respectively). Importantly, in comparison with the patients without dose reduction, the median PFS of patients with the dose reduced by $\geq 1$ was remarkably prolonged (10.43 vs. 5.73 months, respectively). This trial substantiated the activity of anlotinib monotherapy in advanced STS. Since anlotinib was notably effective, it was recommended as a STS treatment by the Chinese Society of Clinical Oncology in 2019 (67). Anlotinib was approved for the second time in China in June 2019 as a second-line treatment for clear cell sarcoma, advanced ASPS, and other STS post-first-line chemotherapies with anthracyclines (68). Tian et al. investigated the effectiveness and safety of apatinib and anlotinib for sarcoma treatment (69). They found that in the treatment of sarcomas, apatinib and anlotinib were effective. Regarding AEs, apatinib was linked to a higher risk of pneumothorax and hair hypopigmentation, while anlotinib was related to a higher rate of hoarseness or pharyngalgia. Wang et al. built a PDX model of malignant fibrous histiocytoma (70) and found that tumor growth can be dose-dependently suppressed by anlotinib or epirubicin. Another study collected medical data of 32 patients with advanced/metastatic STS, retrospectively; the patients received chemotherapy, and anlotinib plus anlotinib maintenance therapy together (71). The results of the study showed that the combination of chemotherapy and anlotinib can largely benefit the survival rate of patients with advanced/metastatic STS, along with good tolerance. The most common grade 3 and 4 AEs were febrile neutropenia (9\%), leukopenia (19\%), thrombocytopenia (3\%), anemia (6\%), anorexia (6\%), vomiting (3\%), and hypertension (6\%); this treatment was generally well-tolerated as a combination therapy. Another study investigated the anti-tumor activity and underlying mechanism of anlotinib in osteosarcoma (56). 
They confirmed that anlotinib inhibited migration and invasion in osteosarcoma cells by suppressing MET and VEGFR2 phosphorylation and downstream signaling pathway activation. Additionally, they showed that hepatocyte growth factor-induced cell migration and invasion as well as VEGF-induced angiogenesis were blocked by anlotinib. The growth and lung metastasis of implanted tumor cells was significantly inhibited by anlotinib in a 143B-Luc orthotopic osteosarcoma model. Tang et al. identified possible mechanism and anti-tumor efficacy of anlotinib in patients with advanced refractory synovial sarcoma (72). They demonstrated that anlotinib may inhibit the proliferation of SS with a new downstream GINS1-regulated network that plays an important role in SS proliferation. Liu et al. analyzed the data of 21 adults with unresectable or metastatic STS who were diagnosed retrospectively. The results indicated that switch maintenance therapy with anlotinib is a promising strategy for the treatment of patients with unresectable or metastatic STS who have received chemotherapy. Liu et al. focused on the detailed absorption, metabolism, and excretion pathways of anlotinib (73). Analysis showed that anlotinib was absorbed rapidly, had a long half-life, and underwent long and extensive hepatic metabolism, which is a good PK profile. The AEs and efficacy were as expected. Li et al. evaluated the function of anlotinib in treating local recurrence or metastatic well-differentiated/dedifferentiated liposarcoma (WDLS/DDLS) (74). This study involved the collection and analysis of baseline and observation indicators. The estimated median PFS was 27.9 weeks, PFS rate at 24 weeks was $58.8 \%$, OS was 56.6 weeks, disease control rate was $64.7 \%$, and no complete response or a partial response was detected. Grade 3/4 AEs occurred in four cases and were managed. This study demonstrated that anlotinib is a potential treatment option for unresectable local recurrence or metastatic WDLS/DDLS.

\section{ANLOTINIB CLINICAL TRIALS FOR MULTIPLE CANCERS}

Clinical trials have assessed the effectiveness and side effects of anlotinib in some advanced solid tumors, including sarcoma, hepatocellular carcinoma, thyroid cancer, squamous cell carcinoma of the esophagus, gastric cancer, small cell lung cancer (SCLC), nonSCLC (NSCLC), metastatic renal cell carcinoma (mRCC), neuroendocrine tumor, and colorectal cancer. Some ongoing trials have attempted to explain the functions of anlotinib in STS, especially the activity of anlotinib in some STS subtypes, such as Ewing's sarcoma, ASPS, synovial sarcoma, and leiomyosarcoma. As a result of low STS incidence, most of these studies are phase III studies. Additionally, some trials are attempting to determine the effectiveness of anlotinib for gastroenteric tumors. In consideration of very limited effective multi-target RTK inhibitors in squamous cell carcinoma of the esophagus and gastric cancer, it is very important to determine the potential roles of anlotinib in these tumors. Moreover, in phase I clinical trials of anlotinib for neuroendocrine carcinoma, there is evidence to suggest a therapeutic effect of anlotinib compared to ideal targeted drugs. Clinical trials are also being conducted to assess the effectiveness of anlotinib in gastrointestinal pancreatic neuroendocrine tumor and SCLC (Table 2).

\section{ONGOING TRIALS}

Given the promising future of anlotinib as a new type of antiangiogenic drug in sarcomas, a large number of related clinical trials are currently registered on the clinicaltrials.gov website (https://clinicaltrials.gov/) (Table 3). Based on the interventional methods, these studies can be divided into the following three types, anlotinib monotherapy, anlotinib plus immunotherapy, and anlotinib plus chemotherapy.

The first type, anlotinib monotherapy, has been conducted to assess its efficacy, safety, and toxicities (NCT03016819, NCT03792542, NCT03890068, NCT04659733, NCT02449343, and NCT03951571). The Second Affiliated Hospital of Zhejiang University School of Medicine has passed a one-arm, multicenter, prospective phase II trial to evaluate anlotinib hydrochloride for patients with advanced STS without chemotherapy, with an estimated enrollment of 44 patients. The clinical trial undertaken by the Sun Yat-sen University Cancer Center Guangzhou is also expected to enroll 48

TABLE 2 | Summary of anlotinib clinical trials for multiple cancers.

\begin{tabular}{|c|c|c|c|}
\hline Regimen & Study type & Enrollment & Population \\
\hline Anlotinib and irinotecan & Phase III & Recruiting & Ewing's sarcoma \\
\hline Anlotinib & Phase $\|/\| \|$ & Recruiting & Soft tissue sarcoma \\
\hline Anlotinib & Phase II & Recruiting & Soft tissue sarcoma \\
\hline Anlotinib & Phase III & Recruiting & Metastatic or advanced alveolar soft part sarcoma, leiomyosarcoma, and synovial sarcoma \\
\hline Anlotinib & Phase ॥ & Recruiting & Colorectal cancer \\
\hline Anlotinib & Phase II & Recruiting & Small cell lung cancer \\
\hline Anlotinib & Phase $\|/\|$ & Recruiting & Gastric cancer \\
\hline Anti-angiogenesis plus EGFR-TKI & Phase II & Recruiting & Non-squamous non-small cell lung cancer \\
\hline Anlotinib & Phase ॥ & Recruiting & Hepatocellular carcinoma \\
\hline Anlotinib & Phase $\|/\| \|$ & Recruiting & Medullary thyroid carcinoma \\
\hline Anlotinib & Phase $\|/\| \|$ & Recruiting & Differentiated thyroid cancer \\
\hline Anlotinib & Phase II & Recruiting & Renal cell carcinoma \\
\hline Anlotinib & Phase II & Recruiting & Esophageal squamous cell carcinoma \\
\hline Anlotinib plus irinotecan & Phase ॥ & Recruiting & Esophageal squamous cell carcinoma \\
\hline Anlotinib & Phase II & Recruiting & Gastroenteropancreatic neuroendocrine tumor G3 \\
\hline
\end{tabular}


TABLE 3 | Selected ongoing trials of anlotinib in sarcoma treatment.

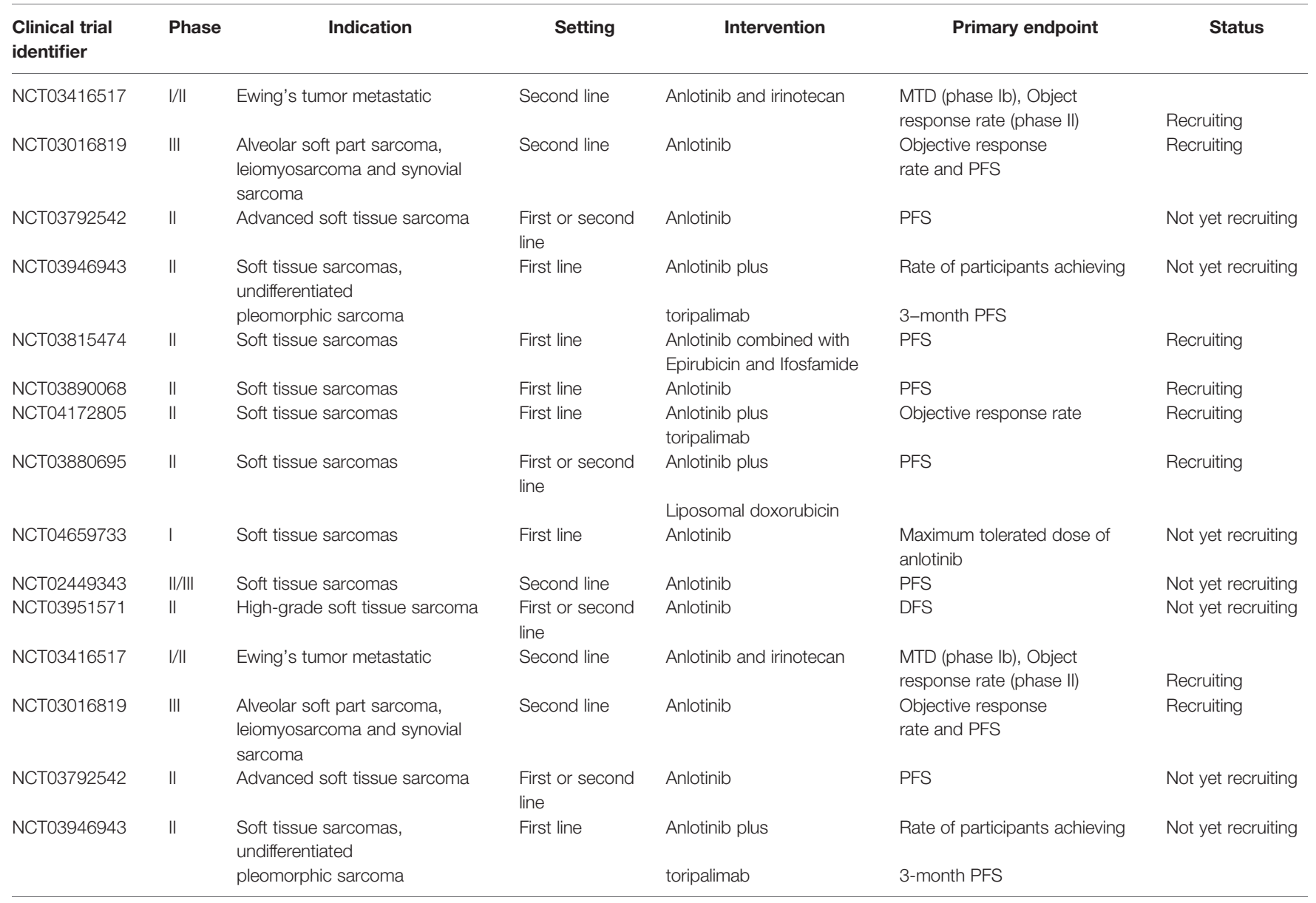

patients with the goal of exploring the safety and efficacy of anlotinib as a maintenance treatment in advanced STS. The Cancer Hospital of Sun Yat-sen Guangzhou has undertaken a prospective randomized double-blind placebo-controlled study to investigate the efficacy and safety of anlotinib hydrochloride in postoperative adjuvant therapy for high-grade STS.

The second type requires researching the anti-neoplastic activity of anlotinib with immunotherapy in sarcomas (NCT03946943 and NCT04172805). The First Hospital of Jilin University has registered a single-arm single-center prospective phase II trial to investigate anlotinib hydrochloride and toripalimab in subjects with unresectable or metastatic undifferentiated pleomorphic sarcoma with an estimated enrollment of 25 patients. The clinical trial registered by Xing Zhang Guangzhou is also expected to enroll 70 patients with the purpose of exploring the safety and efficacy of anlotinib combined with toripalimab in refractory and advanced soft tissue sarcoma.

The third type requires the evaluation of the efficacy and safety of anlotinib combined with chemotherapy in advanced sarcomas (NCT03416517, NCT03815474, and NCT03880695). The Peking University First Hospital has registered a non-randomized phase I/II trial that evaluates anlotinib and irinotecan for advanced Ewing's sarcoma. Overall, 47 patients who failed after standard multimodal therapy participated in the trial. The clinical trial registered by the Liaoning Province Tumor Hospital is also expected to enroll 47 patients with the purpose of exploring the safety and efficacy of anlotinib hydrochloride combined with epirubicin and ifosfamide for patients with locally recurrent or metastatic STS. Peking University Shougang Hospital has registered a one-arm multi-center prospective clinical trial to evaluate the efficacy and safety of anlotinib hydrochloride combined with liposomal doxorubicin in the treatment of locally advanced or metastatic STS.

\section{COMPARISONS OF ANLOTINIB WITH APATINIB AND BEVACIZUMAB}

One of the prerequisites for tumor growth is the generation of internal blood vessels, which can provide sufficient nutrients that provide the material basis for the growth, infiltration, and metastasis of tumor cells $(28,75)$. Therefore, blocking and inhibiting the generation of blood vessels play a vital role in the treatment of malignant tumors. At present, there are at least 20 endogenous angiogenesis inducers known, but VEGF- and VEGFR-mediated signaling pathways play an important role in regulating TA. The VEGFR family includes VEGFR-L, VEGFR-2, 
VEGFR-3, and VEGFR-co-receptor neuraleum L and 2, which regulate mitosis, angiogenesis, and VEGF expression, and in which VEGFR-2 plays an important role $(26,76)$. Moreover, apatinib can also block downstream extracellular signal-related kinase phosphorylation by binding to VEGFR-2, thus, helping treat tumors. The peak blood concentration of apatinib was observed approximately $2.9 \mathrm{~h}$ after oral administration, and the absorption effect was influenced by the order of administration or food (77, 78). Its bioavailability after oral administration was approximately $15 \%$. After four days of administration, approximately $80 \%$ of the drug was excreted through feces and urine, especially feces $(79,80)$. Most adverse reactions are predictable and controllable, and the most common adverse reactions include brothers syndrome, high blood pressure, bleeding, proteinuria, hoarse voice, rash, fatigue, liver damage, diarrhea, and mucosal ulcer rare side effects (81-83). Through changes in suspended medication, dose, and symptomatic treatment, adverse reactions can be controlled and improved.

As a recombinant human monoclonal antibody, bevacizumab is the most-studied anti-angiogenic drug $(84,85)$. The mechanism of action of bevacizumab is that by binding to VEGF, it prevents VEGF from binding to its natural receptor, VEGFR, and inhibits the proliferation and activation of vascular endothelial cells, so as to exert anti-angiogenesis and anti-tumor effects $(86,87)$. VEGF in normal tissue also plays an important role in physiological activity; therefore, the application of bevacizumab bead sheet resistance to inhibit VEGF also leads to some adverse reactions, such as proteinuria, mucosal bleeding (mainly in the nose), and high blood pressure, which is a common adverse reaction. Most cases are mild and self-limiting, requiring only symptomatic treatment $(88,89)$. However, gastrointestinal perforation and thrombosis are serious adverse reactions that require careful handling (89).

As a novel TKI, anlotinib can highly selectively inhibit C-Kit, VEGFR2, PDGFR, FGFR, and other targets, block their downstream signal transduction, play an effective role in anti-TA and tumor growth, and resolve poor effectiveness and toxic reactions. The most common adverse reactions of anlotinib include hand and foot skin reactions, hypertension, fatigue, and lipase elevation, but all adverse reactions are controllable. Anlotinib has the potential for controllable toxicity, long circulation, and broad-spectrum anti-tumor activities, which can be effectively controlled through symptomatic therapy or reduced drug dosage, and is effective in the treatment of a variety of solid tumors.

\section{FUTURE PERSPECTIVES}

Anlotinib was approved in China on May 8, 2018 for the treatment of patients with advanced NSCLC who progressed after treatment with at least two drugs. In the near future, anlotinib may be approved in China for patients with STS who fail to respond to prior traditional treatments. Anlotinib also shows potential as a novel option for treating other solid tumors, such as thyroid cancer and mRCC. Despite certain activities in several cancers, there remain some problems that require further research to solve before its wider application.

First, it is necessary to further predict biomarkers to help detect the most suitable patients for anlotinib treatment.
Although some biomarkers may identify the patients for whom anlotinib will most likely be beneficial, the predictive biomarkers for other types of cancers remain unclear. Further studies are warranted to determine whether anlotinib may be expanded for the treatment of other cancers or be used as a first-line drug, especially a certain subtype of STS.

Additionally, there is synergistic effect when the antiangiogenesis drug ramucirumab is used with chemotherapy (90, 91). In fact, some targeted therapies may also regulate immune responses of the host. Thus, when combined with immunotherapy, the clinical outcome of its application in STS should be further improved. However, most studies have only utilized anlotinib monotherapy, with some exceptions $(92,93)$. Therefore, further studies are warranted for the combination of anlotinib with other treatments. In consideration of the maximum effectiveness of anlotinib for ASPS, it is also necessary to further investigate whether anlotinib may be used as the first-line treatment for these patients. Additionally, the long-term toxicity of anlotinib remains unclear, and thus requires further study. A phase II and III trial has identified some new $\geq$ grade 3 AEs, including hypertriglyceridemia, skin toxicity, neutrophilic granulocytopenia, and hyponatremia, which were not reported in prior clinical trials. Therefore, with further studies on anlotinib, it is necessary to clarify the potential long-term toxicity. Finally, since the studies on anlotinib have only recently started, little is currently known regarding its tumor resistance and its possible mechanism. However, it is of great significance to assess and reverse the drug resistance of anlotinib.

In future studies, individualized therapeutic options should also be developed to overcome drug resistance. For advanced bone and STS, as a result of the heterogeneity characteristics of its pathological and clinical processes, monotherapy-based targeted therapy has not yet been demonstrated. At present, based on the preliminary data of preclinical and clinical studies, anlotinib is promising for the treatment of advanced sarcomas as an antiangiogenesis TKI with notable anti-angiogenesis activity, controllable toxicity, and synergistic anti-tumor efficacy under combination therapy. Meanwhile, some dilemmas need to be addressed, including drug resistance, an appropriate dosage, combined treatment with traditional anti-tumor drugs, sequencing, other anti-angiogenic agents, effective response, and evaluation systems. To obtain satisfactory outcomes with anlotinib as the targeted therapy for patients with advanced sarcomas, these challenges should be studied for single sarcoma types.

\section{CONCLUSIONS}

This review is among few that have addressed the effects of anlotinib on bone and STS. Anlotinib, as a new multi-target RTK inhibitor, has a significant anti-tumor activity for VEGFR signals and inhibition for FGFR 1-3, PDGFR $\alpha$, and c-kit. This is the first approved drug for the third-line treatment of patients with advanced NSCLC in China. With future studies and increased clinical experience, anlotinib is expected to be used for the treatment of other cancers, especially STS. Additionally, with 
good tolerance of anlotinib, most AEs are controllable or reversible through medical intervention. In comparison with other anti-VEGFR TKIs, anlotinib has fewer and milder side effects, especially compared to the thrombocytopenia and neutrophilic granuloaytopenia side effects of sunitinib. Therefore, anlotinib may become a new multi-target RTK.

\section{AUTHOR CONTRIBUTIONS}

The author confirms being the sole contributor of this work and has approved it for publication.

\section{REFERENCES}

1. Callegaro D, Miceli R, Mariani L, Raut CP, Gronchi A. Soft Tissue Sarcoma Nomograms and Their Incorporation Into Practice. Cancer (2017) 123:280220. doi: 10.1002/cncr.30721

2. Thoenen E, Curl A, Iwakuma T. TP53 in Bone and Soft Tissue Sarcomas. Pharmacol Ther (2019) 202:149-64. doi: 10.1016/j.pharmthera.2019.06.010

3. Miwa S, Yamamoto N, Hayashi K, Takeuchi A, Igarashi K, Tsuchiya H. Therapeutic Targets for Bone and Soft-Tissue Sarcomas. Int J Mol Sci (2019) 20:170. doi: 10.3390/ijms20010170

4. Harwood JL, Alexander JH, Mayerson JL, Scharschmidt TJ. Targeted Chemotherapy in Bone and Soft-Tissue Sarcoma. Orthop Clin North Am (2015) 46:587-608. doi: 10.1016/j.ocl.2015.06.011

5. Steffner RJ, Jang ES. Staging of Bone and Soft-tissue Sarcomas. J Am Acad Orthop Surg (2018) 26:e269-78. doi: 10.5435/JAAOS-D-17-00055

6. Jin W. The Role of Tyrosine Kinases as a Critical Prognostic Parameter and Its Targeted Therapies in Ewing Sarcoma. Front Cell Dev Biol (2020) 8:613. doi: $10.3389 /$ fcell.2020.00613

7. Morales E, Olson M, Iglesias F, Dahiya S, Luetkens T, Atanackovic D. Role of Immunotherapy in Ewing Sarcoma. J Immunother Cancer (2020) 8:e00653. doi: 10.1136/jitc-2020-000653

8. Sbaraglia M, Bellan E, Dei Tos AP. The 2020 WHO Classification of Soft Tissue Tumours: News and Perspectives. Pathologica (2020). doi: 10.32074/ 1591-951X-213

9. Nakano K, Takahashi S. Current Molecular Targeted Therapies for Bone and Soft Tissue Sarcomas. Int J Mol Sci (2018) 19:739. doi: 10.3390/ ijms19030739

10. den Hollander D, Van der Graaf WTA, Fiore M, Kasper B, Singer S, Desar IME, et al. Unravelling the Heterogeneity of Soft Tissue and Bone Sarcoma Patients' Health-Related Quality of Life: A Systematic Literature Review With Focus on Tumour Location. ESMO Open (2020) 5:e000914. doi: 10.1136/ esmoopen-2020-000914

11. Kasper B, Hohenberger P. The Challenge of Treating Elderly Patients With Advanced Bone and Soft Tissue Sarcomas. Crit Rev Oncol Hematol (2020) 155:103108. doi: 10.1016/j.critrevonc.2020.103108

12. El-Bared N, Wong P, Wang D. Soft Tissue Sarcoma and Radiation Therapy Advances, Impact on Toxicity. Curr Treat Options Oncol (2015) 16:19. doi: 10.1007/s11864-015-0335-7

13. Spalek MJ, Kozak K, Czarnecka AM, Bartnik E, Borkowska A, Rutkowski P. Neoadjuvant Treatment Options in Soft Tissue Sarcomas. Cancers (Basel) (2020) 12:2061. doi: 10.3390/cancers12082061

14. Di Matteo A, Belloni E, Pradella D, Cappelletto A, Volf N, Zacchigna S, et al. Alternative Splicing in Endothelial Cells: Novel Therapeutic Opportunities in Cancer Angiogenesis. J Exp Clin Cancer Res (2020) 39:275. doi: 10.1186/ s13046-020-01753-1

15. Kasprzak A. Angiogenesis-Related Functions of Wnt Signaling in Colorectal Carcinogenesis. Cancers (Basel) (2020) 12:3601. doi: 10.3390/cancers12123601

16. Menzel L, Hopken UE, Rehm A. Angiogenesis in Lymph Nodes Is a Critical Regulator of Immune Response and Lymphoma Growth. Front Immunol (2020) 11:591741. doi: 10.3389/fimmu.2020.591741

\section{FUNDING}

This work was supported by the Natural Science Foundation of Liaoning Province (2020-MS-058) and Shenyang young and middle-aged scientific and technological innovation talent support plan (RC190456).

\section{ACKNOWLEDGMENTS}

The author thanks the generous support of the Liaoning Cancer Hospital \& Institute (Shenyang).

17. Tan HW, Xu YM, Qin SH, Chen GF, Lau ATY. Epigenetic Regulation of Angiogenesis in Lung Cancer. J Cell Physiol (2020) 236:3194-206. doi: $10.1002 /$ jcp.30104

18. Yetkin-Arik B, Kastelein AW, Klaassen I, Jansen C, Latul YP, Vittori M, et al. Angiogenesis in Gynecological Cancers and the Options for Anti-Angiogenesis Therapy. Biochim Biophys Acta Rev Cancer (2020) 1875:188446. doi: 10.1016/ j.bbcan.2020.188446

19. Jiang X, Wang J, Deng X, Xiong F, Zhang S, Gong Z, et al. The Role of Microenvironment in Tumor Angiogenesis. J Exp Clin Cancer Res (2020) 39:204. doi: 10.1186/s13046-020-01709-5

20. de Heer EC, Jalving M, Harris AL. Hifs, Angiogenesis, and Metabolism: Elusive Enemies in Breast Cancer. J Clin Invest (2020) 130:5074-87. doi: 10.1172/JCI137552

21. Zhu XD, Tang ZY, Sun HC. Targeting Angiogenesis for Liver Cancer: Past, Present, and Future. Genes Dis (2020) 7:328-35. doi: 10.1016/j.gendis.2020. 03.010

22. Campochiaro PA. Low Risk to Retina From Sustained Suppression of VEGF. J Clin Invest (2019) 129:3029-31. doi: 10.1172/JCI129861

23. Choueiri TK, Kaelin WG Jr. Targeting the HIF2-VEGF Axis in Renal Cell Carcinoma. Nat Med (2020) 26:1519-30. doi: 10.1038/s41591-020-1093-Z

24. Khodarahmian M, Amidi F, Moini A, Kashani L, Salahi E, Danaii-Mehrabad S, et al. A Randomized Exploratory Trial to Assess the Effects of Resveratrol on VEGF and TNF-alpha 2 Expression in Endometriosis Women. J Reprod Immunol (2020) 143:103248. doi: 10.1016/j.jri.2020.103248

25. Shaik F, Cuthbert GA, Homer-Vanniasinkam S, Muench SP, Ponnambalam S, Harrison MA. Structural Basis for Vascular Endothelial Growth Factor Receptor Activation and Implications for Disease Therapy. Biomolecules (2020) 10:1673. doi: 10.3390/biom10121673

26. Campochiaro PA, Akhlaq A. Sustained Suppression of VEGF for Treatment of Retinal/Choroidal Vascular Diseases. Prog Retin Eye Res (2020) 100921 doi: 10.1016/j.preteyeres.2020.100921

27. Wang X, Bove AM, Simone G, Ma B. Molecular Bases of VEGFR-2-Mediated Physiological Function and Pathological Role. Front Cell Dev Biol (2020b) 8:599281. doi: 10.3389/fcell.2020.599281

28. Yang QK, Chen T, Wang SQ, Zhang XJ, Yao ZX. Apatinib as Targeted Therapy for Advanced Bone and Soft Tissue Sarcoma: A Dilemma of Reversing Multidrug Resistance While Suffering Drug Resistance Itself. Angiogenesis (2020b) 23:279-98. doi: 10.1007/s10456-020-09716-y

29. Han B, Li K, Wang Q, Zhang L, Shi J, Wang Z, et al. Effect of Anlotinib as a Third-Line or Further Treatment on Overall Survival of Patients With Advanced Non-Small Cell Lung Cancer: The ALTER 0303 Phase 3 Randomized Clinical Trial. JAMA Oncol (2018) 4:1569-75. doi: 10.1001/ jamaoncol.2018.3039

30. He C, Wu T, Hao Y. Anlotinib Induces Hepatocellular Carcinoma Apoptosis and Inhibits Proliferation Via Erk and Akt Pathway. Biochem Biophys Res Commun (2018) 503:3093-99. doi: 10.1016/j.bbrc.2018.08.098

31. Xie C, Wan X, Quan H, Zheng M, Fu L, Li Y, et al. Preclinical Characterization of Anlotinib, a Highly Potent and Selective Vascular Endothelial Growth Factor Receptor-2 Inhibitor. Cancer Sci (2018) 109:1207-19. doi: 10.1111/ cas. 13536 
32. Guo W, Chen S, Wu Z, Zhuang W, Yang J. Efficacy and Safety of Transarterial Chemoembolization Combined With Anlotinib for Unresectable Hepatocellular Carcinoma: A Retrospective Study. Technol Cancer Res Treat (2020) 19:1533033820965587. doi: 10.1177/1533033820965587

33. Liu J, Li S, Zhang S, Yang C, Zhang L, Zhang B, et al. Pretreatment Prognostic Nutritional Index Is a Prognostic Marker for Extensive-Stage Small Cell Lung Cancer Patients Treated With Anlotinib. J Thorac Dis (2020a) 12:5765-73. doi: $10.21037 /$ jtd-20-755

34. Ni J, Cheng X, Chen J, Guo W, Dai Z. Anlotinib as Exploratory Therapy for Platinum-Resistant Ovarian Cancer: A Retrospective Study on Efficacy and Safety. Onco Targets Ther (2020) 13:9857-63. doi: 10.2147/OTT.S268613

35. Shi J, Zhang Y, Wang J, Li J, Li Z. Anlotinib Combined With Chemoradiotherapy Exhibits Significant Therapeutic Efficacy in Esophageal Squamous Cell Carcinoma. Front Oncol (2020) 10:995. doi: 10.3389/ fonc. 2020.00995

36. Xie XH, Wang F, Lin XQ, Qin YY, Xie ZH, Zhang JX, et al. Anlotinib Plus s-1 for Patients With EGFR Mutation-Negative Advanced Squamous Cell Lung Cancer With PS Scores of 2-3 After Progression of Second-Line or Later-Line Treatment. Cancer Manag Res (2020) 12:12709-14. doi: 10.2147/ CMAR.S278068

37. Zhong Y, Wei Q, Lu Y, Tang X, Wang Z, Chen L. Efficacy and Safety of Anlotinib in Patients With Advanced non-Small Cell Lung Cancer. J Thorac Dis (2020) 12:6016-22. doi: 10.21037/jtd-20-2855

38. Zhou W, Gao Y, Tong Y, Wu Q, Zhou Y, Li Y. Anlotinib Enhances the Antitumor Activity of Radiofrequency Ablation on Lung Squamous Cell Carcinoma. Pharmacol Res (2020) 164:105392. doi: 10.1016/j.phrs.2020. 105392

39. Sun Y, Niu W, Du F, Du C, Li S, Wang J, et al. Safety, Pharmacokinetics, and Antitumor Properties of Anlotinib, an Oral Multi-Target Tyrosine Kinase Inhibitor, in Patients With Advanced Refractory Solid Tumors. J Hematol Oncol (2016) 9:105. doi: 10.1186/s13045-016-0332-8

40. Shen G, Zheng F, Ren D, Du F, Dong Q, Wang Z, et al. Anlotinib: A Novel Multi-Targeting Tyrosine Kinase Inhibitor in Clinical Development. J Hematol Oncol (2018) 11:120. doi: 10.1186/s13045-018-0664-7

41. Qin T, Liu Z, Wang J, Xia J, Liu S, Jia Y, et al. Anlotinib Suppresses Lymphangiogenesis and Lymphatic Metastasis in Lung Adenocarcinoma Through a Process Potentially Involving VEGFR-3 Signaling. Cancer Biol Med (2020) 17:753-67. doi: 10.20892/j.issn.2095-3941.2020.0024

42. Wang Y, Liang D, Chen J, Chen H, Fan R, Gao Y, et al. Targeted Therapy With Anlotinib for a Patient With an Oncogenic Fgfr3-Tacc3 Fusion and Recurrent Glioblastoma. Oncologist (2020c) 26:173-7. doi: 10.1002/onco.13530

43. Yang L, Zhou X, Sun J, Lei Q, Wang Q, Pan D, et al. Reactive Oxygen Species Mediate Anlotinib-Induced Apoptosis Via Activation of Endoplasmic Reticulum Stress in Pancreatic Cancer. Cell Death Dis (2020a) 11:766. doi: 10.1038/s41419-020-02938-4

44. Lin B, Song X, Yang D, Bai D, Yao Y, Lu N. Anlotinib Inhibits Angiogenesis Via Suppressing the Activation of VEGFR2, Pdgfr $\beta$ and FGFR1. Gene (2018b) 654:77-86. doi: 10.1016/j.gene.2018.02.026

45. Lu C, Zhang Q, Zhang H, Li X, Jiang Q, Yao J. A Small Molecular MultiTargeting Tyrosine Kinase Inhibitor, Anlotinib, Inhibits Pathological Ocular Neovascularization. BioMed Pharmacother (2021) 138:111493. doi: 10.1016/ j.biopha.2021.111493

46. Lin B, Song X, Yang D, Bai D, Yao Y, Lu N. Anlotinib Inhibits Angiogenesis Via Suppressing the Activation of VEGFR2, Pdgfrbeta and FGFR1. Gene (2018a) 654:77-86. doi: 10.1016/j.gene.2018.02.026

47. Taurin S, Yang CH, Reyes M, Cho S, Coombs DM, Jarboe EA, et al. Endometrial Cancers Harboring Mutated Fibroblast Growth Factor Receptor 2 Protein Are Successfully Treated With a New Small Tyrosine Kinase Inhibitor in an Orthotopic Mouse Model. Int J Gynecol Cancer (2018) 28:152-60. doi: 10.1097/IGC.0000000000001129

48. Zhong CC, Chen F, Yang JL, Jia WW, Li L, Cheng C, et al. Pharmacokinetics and Disposition of Anlotinib, an Oral Tyrosine Kinase Inhibitor, in Experimental Animal Species. Acta Pharmacol Sin (2018) 39:1048-63. doi: 10.1038/aps.2017.199

49. Sun W, Wang Z, Chen R, Huang C, Sun R, Hu X, et al. Influences of Anlotinib on Cytochrome P450 Enzymes in Rats Using a Cocktail Method. BioMed Res Int (2017) 2017:3619723. doi: 10.1155/2017/3619723
50. van Erp NP, Gelderblom H, Guchelaar HJ. Clinical Pharmacokinetics of Tyrosine Kinase Inhibitors. Cancer Treat Rev (2009) 35:692-706. doi: 10.1016/j.ctrv.2009.08.004

51. Gore ME, Szczylik C, Porta C, Bracarda S, Bjarnason GA, Oudard S, et al. Safety and Efficacy of Sunitinib for Metastatic Renal-Cell Carcinoma: An Expanded-Access Trial. Lancet Oncol (2009) 10:757-63. doi: 10.1016/S14702045(09)70162-7

52. Stadler WM, Figlin RA, McDermott DF, Dutcher JP, Knox JJ, Miller WHJr, et al. Safety and Efficacy Results of the Advanced Renal Cell Carcinoma Sorafenib Expanded Access Program in North America. Cancer (2010) 116:1272-80. doi: 10.1002/cncr.24864

53. Beck J, Procopio G, Bajetta E, Keilholz U, Negrier S, Szczylik C, et al. Final Results of the European Advanced Renal Cell Carcinoma Sorafenib (EuARCCS) Expanded-Access Study: A Large Open-Label Study in Diverse Community Settings. Ann Oncol (2011) 22:1812-23. doi: 10.1093/annonc/ mdq651

54. Qi WX, Shen Z, Tang LN, Yao Y. Risk of Arterial Thromboembolic Events With Vascular Endothelial Growth Factor Receptor Tyrosine Kinase Inhibitors: An Up-to-Date Meta-Analysis. Crit Rev Oncol Hematol (2014) 92:71-82. doi: 10.1016/j.critrevonc.2014.04.004

55. Liang L, Hui K, Hu C, Wen Y, Yang S, Zhu P, et al. Autophagy Inhibition Potentiates the Anti-Angiogenic Property of Multikinase Inhibitor Anlotinib Through JAK2/STAT3/VEGFA Signaling in Non-Small Cell Lung Cancer Cells. J Exp Clin Cancer Res (2019) 38:71. doi: 10.1186/s13046-019-1093-3

56. Wang G, Sun M, Jiang Y, Zhang T, Sun W, Wang H, et al. Anlotinib, a Novel Small Molecular Tyrosine Kinase Inhibitor, Suppresses Growth and Metastasis Via Dual Blockade of VEGFR2 and MET in Osteosarcoma. Int $J$ Cancer (2019) 145:979-93. doi: 10.1002/ijc.32180

57. Ruan X, Shi X, Dong Q, Yu Y, Hou X, Song X, et al. Antitumor Effects of Anlotinib in Thyroid Cancer. Endocr Relat Cancer (2019) 26:153-64. doi: 10.1530/ERC-17-0558

58. Turkington RC, Longley DB, Allen WL, Stevenson L, McLaughlin K, Dunne PD, et al. Fibroblast Growth Factor Receptor 4 (FGFR4): A Targetable Regulator of Drug Resistance in Colorectal Cancer. Cell Death Dis (2014) 5: e1046. doi: 10.1038/cddis.2014.10

59. Saito S, Morishima K, Ui T, Hoshino H, Matsubara D, Ishikawa S, et al. The Role of HGF/MET and FGF/FGFR in Fibroblast-Derived Growth Stimulation and Lapatinib-Resistance of Esophageal Squamous Cell Carcinoma. BMC Cancer (2015) 15:82. doi: 10.1186/s12885-015-1065-8

60. Sugimoto K, Miyata Y, Nakayama T, Saito S, Suzuki R, Hayakawa F, et al. Fibroblast Growth Factor-2 Facilitates the Growth and Chemo-Resistance of Leukemia Cells in the Bone Marrow by Modulating Osteoblast Functions. Sci Rep (2016) 6:30779. doi: 10.1038/srep30779

61. Jang HS, Woo SR, Song KH, Cho H, Chay DB, Hong SO, et al. API5 Induces Cisplatin Resistance Through FGFR Signaling in Human Cancer Cells. Exp Mol Med (2017) 49:e374. doi: 10.1038/emm.2017.130

62. Wilding CP, Elms ML, Judson I, Tan AC, Jones RL, Huang PH. The Landscape of Tyrosine Kinase Inhibitors in Sarcomas: Looking Beyond Pazopanib. Expert Rev Anticancer Ther (2019) 19:971-91. doi: 10.1080/ 14737140.2019.1686979

63. Agulnik M, Yarber JL, Okuno SH, von Mehren M, Jovanovic BD, Brockstein BE, et al. An Open-Label, Multicenter, Phase II Study of Bevacizumab for the Treatment of Angiosarcoma and Epithelioid Hemangioendotheliomas. Ann Oncol (2013) 24:257-63. doi: 10.1093/annonc/mds237

64. Tap WD, Jones RL, Van Tine BA, Chmielowski B, Elias AD, Adkins D, et al. Olaratumab and Doxorubicin Versus Doxorubicin Alone for Treatment of Soft-Tissue Sarcoma: An Open-Label Phase $1 \mathrm{~b}$ and Randomised Phase 2 Trial. Lancet (2016) 388:488-97. doi: 10.1016/S0140-6736(16)30587-6

65. Sleijfer S, Ray-Coquard I, Papai Z, Le Cesne A, Scurr M, Schoffski P, et al. Pazopanib, a Multikinase Angiogenesis Inhibitor, in Patients With Relapsed or Refractory Advanced Soft Tissue Sarcoma: A Phase II Study From the European Organisation for Research and Treatment of Cancer-Soft Tissue and Bone Sarcoma Group (EORTC Study 62043). J Clin Oncol (2009) 27:3126-32. doi: 10.1200/JCO.2008.21.3223

66. Stacchiotti S, Negri T, Zaffaroni N, Palassini E, Morosi C, Brich S, et al. Sunitinib in Advanced Alveolar Soft Part Sarcoma: Evidence of a Direct Antitumor Effect. Ann Oncol (2011) 22:1682-90. doi: 10.1093/annonc/mdq644 
67. Sternberg CN, Davis ID, Mardiak J, Szczylik C, Lee E, Wagstaff J, et al. Pazopanib in Locally Advanced or Metastatic Renal Cell Carcinoma: Results of a Randomized Phase III Trial. J Clin Oncol (2010) 28:1061-8. doi: 10.1200/ JCO.2009.23.9764

68. Motzer RJ, Hutson TE, Tomczak P, Michaelson MD, Bukowski RM, Rixe O, et al. Sunitinib Versus Interferon Alfa in Metastatic Renal-Cell Carcinoma. N Engl J Med (2007) 356:115-24. doi: 10.1056/NEJMoa065044

69. Tian Z, Liu H, Zhang F, Li L, Du X, Li C, et al. Retrospective Review of the Activity and Safety of Apatinib and Anlotinib in Patients With Advanced Osteosarcoma and Soft Tissue Sarcoma. Invest New Drugs (2020) 38:1559-69. doi: 10.1007/s10637-020-00912-7

70. Wang ZM, Zhang SL, Yang H, Zhuang RY, Guo X, Tong HX, et al. Efficacy and Safety of Anlotinib, a Multikinase Angiogenesis Inhibitor, in Combination With Epirubicin in Preclinical Models of Soft Tissue Sarcoma. Cancer Med (2020d) 9:3344-52. doi: 10.1002/cam4.2941

71. Wang HY, Chu JF, Zhang P, Wang JQ, Yan Z, Yao SN, et al. Safety and Efficacy of Chemotherapy Combined With Anlotinib Plus Anlotinib Maintenance in Chinese Patients With Advanced/Metastatic Soft Tissue Sarcoma. Onco Targets Ther (2020a) 13:1561-8. doi: 10.2147/OTT.S235349

72. Tang L, Yu W, Wang Y, Li H, Shen Z. Anlotinib Inhibits Synovial Sarcoma by Targeting GINS1: A Novel Downstream Target Oncogene in Progression of Synovial Sarcoma. Clin Transl Oncol (2019) 21:1624-33. doi: 10.1007/s12094019-02090-2

73. Liu Y, Liu L, Liu L, Wang T, Guo L, Wang Y, et al. A Phase I Study Investigation of Metabolism, and Disposition of [(14)C]-Anlotinib After an Oral Administration in Patients With Advanced Refractory Solid Tumors. Cancer Chemother Pharmacol (2020b) 85:907-15. doi: 10.1007/s00280-02004062-8

74. Li ZK, Liu J, Deng YT, Jiang Y. Efficacy and Safety of Anlotinib in Patients With Unresectable or Metastatic Well-Differentiated/Dedifferentiated Liposarcoma: A Single-Center Retrospective Study. Anticancer Drugs (2021) 32:210-4. doi: 10.1097/CAD.0000000000001023

75. Fathi Maroufi N, Rashidi MR, Vahedian V, Akbarzadeh M, Fattahi A, Nouri M. Therapeutic Potentials of Apatinib in Cancer Treatment: Possible Mechanisms and Clinical Relevance. Life Sci (2020) 241:117106. doi: $10.1016 /$ j.lfs.2019.117106

76. Ntellas P, Mavroeidis L, Gkoura S, Gazouli I, Amylidi AL, Papadaki A, et al. Old Player-New Tricks: Non Angiogenic Effects of the VEGF/VEGFR Pathway in Cancer. Cancers (Basel) (2020) 12:3145. doi: 10.3390/ cancers 12113145

77. Geng R, Song L, Li J, Zhao L. The Safety of Apatinib for the Treatment of Gastric Cancer. Expert Opin Drug Saf (2018) 17:1145-50. doi: 10.1080/ 14740338.2018.1535592

78. Jin M, Cai J, Wang X, Zhang T, Zhao Y. Successful Maintenance Therapy With Apatinib Inplatinum-Resistant Advanced Ovarian Cancer and Literature Review. Cancer Biol Ther (2018) 19:1088-92. doi: 10.1080/ 15384047.2018.1491500

79. Li CM, Liu ZC, Bao YT, Sun XD, Wang LL. Extraordinary Response of Metastatic Pancreatic Cancer to Apatinib After Failed Chemotherapy: A Case Report and Literature Review. World J Gastroenterol (2017) 23:7478-88. doi: 10.3748/wjg.v23.i41.7478

80. Zhao D, Hou H, Zhang X. Progress in the Treatment of Solid Tumors With Apatinib: A Systematic Review. Onco Targets Ther (2018) 11:4137-47. doi: 10.2147/OTT.S172305

81. Cheng H, Sun A, Guo Q, Zhang Y. Efficacy and Safety of Apatinib Combined With Chemotherapy for the Treatment of Advanced Gastric Cancer in the
Chinese Population: A Systematic Review and Meta-Analysis. Drug Des Devel Ther (2018) 12:2173-83. doi: 10.2147/DDDT.S170678

82. Li F, Liao Z, Zhang C, Zhao J, Xing R, Teng S, et al. Apatinib as Targeted Therapy for Sarcoma. Oncotarget (2018) 9:24548-60. doi: 10.18632/ oncotarget. 24647

83. Scott LJ. Apatinib: A Review in Advanced Gastric Cancer and Other Advanced Cancers. Drugs (2018) 78:747-58. doi: 10.1007/s40265-018-0903-9

84. Dang A, Jagan Mohan Venkateshwara Rao P, Kishore R, Vallish BN. Real World Safety of Bevacizumab in Cancer Patients: A Systematic Literature Review of Case Reports. Int J Risk Saf Med (2020). doi: 10.3233/JRS-194051

85. Taieb J, Aranda E, Raouf S, Dunn H, Arnold D. Clinical and Regulatory Considerations for the Use of Bevacizumab Biosimilars in Metastatic Colorectal Cancer. Clin Colorectal Cancer (2020) 20:42-51. doi: 10.1016/ j.clcc.2020.10.005

86. Plummer C, Michael A, Shaikh G, Stewart M, Buckley L, Miles T, et al. Expert Recommendations on the Management of Hypertension in Patients With Ovarian and Cervical Cancer Receiving Bevacizumab in the UK. Br J Cancer (2019) 121:109-16. doi: 10.1038/s41416-019-0481-y

87. Garcia J, Hurwitz HI, Sandler AB, Miles D, Coleman RL, Deurloo R, et al. Bevacizumab (Avastin(R)) in Cancer Treatment: A Review of 15 Years of Clinical Experience and Future Outlook. Cancer Treat Rev (2020) 86:102017. doi: $10.1016 / j . c t r v .2020 .102017$

88. Marchetti C, Muzii L, Romito A, Benedetti Panici P. First-Line Treatment of Women With Advanced Ovarian Cancer: Focus on Bevacizumab. Onco Targets Ther (2019) 12:1095-103. doi: 10.2147/OTT.S155425

89. Zhuang H, Shi S, Yuan Z, Chang JY. Bevacizumab Treatment for Radiation Brain Necrosis: Mechanism, Efficacy and Issues. Mol Cancer (2019) 18:21. doi: 10.1186/s12943-019-0950-1

90. Garon EB, Ciuleanu TE, Arrieta O, Prabhash K, Syrigos KN, Goksel T, et al. Ramucirumab Plus Docetaxel Versus Placebo Plus Docetaxel for Second-Line Treatment of Stage IV Non-Small-Cell Lung Cancer After Disease Progression on Platinum-Based Therapy (REVEL): A Multicentre, Double-Blind, Randomised Phase 3 Trial. Lancet (2014) 384:665-73. doi: 10.1016/S01406736(14)60845-X

91. Wilke H, Muro K, Van Cutsem E, Oh SC, Bodoky G, Shimada Y, et al. Ramucirumab Plus Paclitaxel Versus Placebo Plus Paclitaxel in Patients With Previously Treated Advanced Gastric or Gastro-Oesophageal Junction Adenocarcinoma (RAINBOW): A Double-Blind, Randomised Phase 3 Trial. Lancet Oncol (2014) 15:1224-35. doi: 10.1016/S1470-2045(14)70420-6

92. Vanneman M, Dranoff G. Combining Immunotherapy and Targeted Therapies in Cancer Treatment. Nat Rev Cancer (2012) 12:237-51. doi: $10.1038 / \mathrm{nrc} 3237$

93. Hughes PE, Caenepeel S, Wu LC. Targeted Therapy and Checkpoint Immunotherapy Combinations for the Treatment of Cancer. Trends Immunol (2016) 37:462-76. doi: 10.1016/j.it.2016.04.010

Conflict of Interest: The author declares that the research was conducted in the absence of any commercial or financial relationships that could be construed as a potential conflict of interest.

Copyright () $2021 \mathrm{Li}$. This is an open-access article distributed under the terms of the Creative Commons Attribution License (CC BY). The use, distribution or reproduction in other forums is permitted, provided the original author(s) and the copyright owner(s) are credited and that the original publication in this journal is cited, in accordance with accepted academic practice. No use, distribution or reproduction is permitted which does not comply with these terms. 